\title{
A Grey Transport Acceleration Method for Time-Dependent Radiative Transfer Problems
}

\author{
EDWARD LARSEN \\ Department of Nuclear Engineering, University of Michigan, \\ Ann Arbor, Michigan 48109
}

Received September 1, 1987; revised November 30, 1987

\begin{abstract}
A new iterative method for solving the time-dependent multifrequency radiative transfer equations is described. The method is applicable to semi-implicit time discretizations that generate a linear steady-state multifrequency transport problem with pseudo-scattering within each time step. The standard "lambda" iteration method is shown to often converge slowly for such problems, and the new grey transport acceleration (GTA) method, based on accelerating the lambda method by employing a grey, or frequency-independent transport equation, is developed. The GTA method is shown, theoretically by an iterative Fourier analysis, and experimentally by numerical calculations, to converge significantly faster than the lambda method. In addition, the GTA method is conceptually simple to implement for general differencing schemes, on cither Eulerian or Lagrangian meshes. (c) 1988 Academic Press, Iatc.
\end{abstract}

\section{INTRODUCTION}

The equations of thermal radiative transfer describe the transport, absorption, and emission of photons within a physical material. As photons propagate, they are absorbed and emitted by a Planckian distribution; in this process, the material temperature can change, thereby changing the shape of the Planckian. This transport process is inherently nonlinear, and because of this and other properties of the equations of radiative transfer, standard numerical methods frequently tend to work rather poorly. In particular, when the coupling between the material and radiation is strong, as occurs when the material opacity or the time step is large, standard iterative methods for solving the radiative transfer equations generally converge very slowly.

In this article we describe, analyze, and present numerical test results from a new iterative grey transport acceleration (GTA) method for solving time-dependent thermal radiative transfer problems. The GTA method is conceptually simple and easy to implement, for problems with general differencing schemes on Eulerian or Lagrangian meshes. The method is specifically designed for problems which are time-differenced in a semi-implicit manner, yielding a transport equation with a "pseudo-scattering" $[1,2]$ term. In large-scale problems this equation must be solved iteratively, and as mentioned above, standard iterative methods converge very slowly when the time step or the opacity is large. To accelerate this 
convergence by the GTA method, one group-collapses the frequency-dependent transport equation into a grey transport equation and introduces this new equation into the lambda iteration algorithm. The derivation of the GTA method is the subject of this article.

Other iterative acceleration methods have been proposed for time-dependent radiative transfer problems. In particular, Auer [3] has recently reviewed a number of methods that have been developed in the astrophysical community. However, this work is oriented to one-dimensional geometries, and our concern here is with methods that are potentially applicable in multidimensional geometries for general types of meshes.

The diffusion-synthetic acceleration (DSA) algorithm [2], originally developed for neutronics problems $[4,5]$, is applicable in multidimensional geometries. This is a nested iterative algorithm in which the convergence of the multifrequency transport equation with pseudo-scattering is accelerated by the solution of a multifrequency diffusion equation, and the convergence of the multifrequency diffusion equation is accelerated by the solution of a grey diffusion equation. When it is applicable, the DSA method is more efficient than the new GTA method. However, the DS $\Lambda$ method is complicated to implement, and stability difficulties, caused by accelerating the convergence of a transport equation with a diffusion equation, have, to this date, restricted the 2-D applications of the method to rectangular (Eulerian) spatial grids $[2,4]$, with the diamond difference spatial discretization method $[2,4,6,7]$. The GTA method, on the other hand, is much simpler to implement and is applicable to general differencing schemes on general types of meshes. Thus, although the DSA method is more efficient when it can be used, the GTA method is more easily implemented and wider in its range of applicability.

We reiteratc that the DSA method contains, at the "inner" iteration level, an algorithm in which the convergence of a multifrequency diffusion equation is accelerated by solving a grey diffusion equation. This "multifrequency-grey" (MFG) iteration $[2,8,9]$ is conceptually very similar to the GTA method considered here, except that with GTA, the underlying equations are transport, rather than diffusion. Also, the GTA method considered in this paper is linear, in the sense that the terms that make our grey transport equation consistent with the multifrequency equation are additive. The MFG method can be formulated eiher linearly or nonlinearly [2]; in the latter case, the consistency terms are multiplicative. The nonlinear formulation, which can also be applied to the transport equation, is described in more detail in Section VIII.

A summary of the remainder of this paper follows. In Section II we introduce notation, formulate two semi-implicit time differencing schemes, and for each scheme derive linear, steady-state, multifrequency transport equations with pseudoscattering within each time step. In Secton III we describe the lambda iteration method for these equations and prove theoretically, by an iterative Fourier analysis, that this method converges slowly when either the time step or the material opacity is large. In Sections IV and V we develop the GTA method, and in Secton VI, we show via another Fourier analysis, that its convergence properties are a significant 
improvement over those of lambda iteration. In Section VII we describe some numerical results from an implementation of the GTA method in a test code, and we conclude with a brief discussion in Section VIII.

\section{The Radiative Transfer Equations}

In the absence of material motion, scattering, interior sources, and heat conduction, the equations of thermal radiative transfer $[2,10,11]$ consist of the equation of transfer

$$
\frac{1}{c} \frac{\partial I}{\partial t}+\mathbf{\Omega} \cdot \nabla I=\sigma(B-I)
$$

and the energy balance equation

$$
c_{v} \frac{\partial T}{\partial t}=\iint \sigma(I-B) d \Omega^{\prime} d v^{\prime}
$$

together with the initial conditions

$$
\begin{gathered}
I(\mathbf{x}, \mathbf{\Omega}, v, 0)=I_{i}(\mathbf{x}, \mathbf{\Omega}, v) \\
T(\mathbf{x}, 0)=T_{i}(\mathbf{x})
\end{gathered}
$$

and the boundary condition

$$
I(\mathbf{x}, \mathbf{\Omega}, v, t)=I_{b}(\mathbf{x}, \boldsymbol{\Omega}, v, t), \quad \mathbf{x} \in \partial D, \quad \mathbf{\Omega}: \mathbf{n}<0 .
$$

The independent variables in these equations are $\mathbf{x}$ (position), $\boldsymbol{\Omega}$ (direction), $v$ (frequency), and $t$ (time). The unknowns are the specific photon intensity $I(\mathbf{x}, \Omega, v, t)$ and the material temperature $T(\mathbf{x}, t)$. The known expressions are the opacity $\sigma(v, T)$, the heat capacity $c_{v}(T)$, and the Planck function

$$
B(v, T)=\frac{2 h v^{3}}{c^{2}}\left(e^{h v / k T}-1\right)^{-1}
$$

where $c$ is the speed of light, $h$ is Planck's constant, and $k$ is Boltzmann's constant. Later we shall use the following explicit forms for $c_{v}$ and $\sigma$,

$$
\begin{aligned}
c_{v}(T) & =c_{v}=\text { constant } \\
\sigma(v, T) & =\frac{\gamma}{(h v)^{3}}\left(1-e^{-h v / k T}\right)
\end{aligned}
$$

with $\gamma=$ constant. 
Let us time-discretize Eqs. (2.1) and (2.2) in the following semi-implicit way:

$$
\begin{gathered}
\frac{I_{n+1}-I_{n}}{c \Delta t_{n}}+\boldsymbol{\Omega} \cdot \nabla I_{n+1}=\sigma_{n}\left(b_{n+1}-I_{n+1}\right), \\
c_{v, n} \frac{T_{n+1}-T_{n}}{\Delta t_{n}}=\iint \sigma_{n}\left(I_{n+1}-b_{n+1}\right) d \Omega^{\prime} d v^{\prime} .
\end{gathered}
$$

Here the subscripts refer to the time at which the indicated quantity is evaluated. Thus, $\sigma$ and $c_{v}$ are evaluated at the old time step, and an approximation $b$ of the Planck function is evaluated at the new time step. It is not feasable to implicitly set $b_{n+1}=B_{n+1}=B\left(v, T_{n+1}\right)$, because Eqs. (2.8) and (2.9) become nonlinear. Likewise, it is not efficient to explicitly set $b_{n+1}=B_{n}$, because then extremely small time steps are required to obtain accurate solutions. Therefore, one compromises and takes $b_{n+1}$ to be a suitable approximation to $B_{n+1}$. We now describe two such approximations.

The "nonlinear" semi-implicit method, which is based on the implicit Monte Carlo method [1], uses the identity

$$
\Phi_{n}=4 \pi \int B_{n} d v^{\prime}=a c T_{n}^{4},
$$

where $a$ is the radiation constant [10], and takes

$$
b_{n+1}=\frac{B\left(v, T_{n}\right)}{\Phi_{n}} \phi_{n+1},
$$

where $\phi_{n+1}$ is an approximation to $\Phi_{n+1}$, derived as follows. Noting that

$$
\frac{\partial \Phi}{\partial T}=4 a c T^{3}=\frac{4 \Phi}{T}
$$

so

$$
c_{v} \frac{\partial T}{\partial t}=c_{v} \frac{\partial T}{\partial \Phi} \frac{\partial \Phi}{\partial t}=\frac{c_{v} T}{4 \Phi} \frac{\partial \Phi}{\partial t} \equiv \frac{1}{\beta} \frac{\partial \Phi}{\partial t}
$$

we can rewrite Eq. (2.2) as

$$
\frac{1}{\beta} \frac{\partial \Phi}{\partial t}=\iint \sigma(I-B) d \Omega^{\prime} d v^{\prime}
$$

and time-difference it in the form

$$
\frac{\phi_{n+1}-\Phi_{n}}{\beta_{n} \Delta t_{n}}=\iint \sigma_{n}\left(I_{n+1}-\frac{B_{n}}{\Phi_{n}} \phi_{n+1}\right) d \Omega^{\prime} d v^{\prime}
$$


Solving this equation for $\phi_{n+1}$ and introducing the result into Eq. (2.11), we obtain

$$
b_{n+1}=B_{n} \frac{\Phi_{n}+\beta_{n} \Delta t_{n} \iint \sigma_{n} I_{n+1} d Q^{\prime} d v^{\prime}}{\Phi_{n}+\beta_{n} \Delta t_{n} \iint \sigma_{n} B_{n} d \Omega^{\prime} d v^{\prime}} .
$$

The "nonlinear" semi-implicit method now consists of Eqs. (2.8), (2.9), and (2.16). For this method, the above approximation $b_{n, 1}$ of $B_{n ! 1}$ is positive, but it has an $O(\Delta T)$ error. We refer to this as the "nonlinear" method because the correction to $B_{n}$ in Eq. (2.16) is multiplicative. However, the resulting equations yield linear relationships among $I_{n+1}$ and $T_{n+1}$.

The "linear" semi-implicit method takes, simply,

$$
b_{n+1}=B\left(v, T_{n}\right)+\left(T_{n+1}-T_{n}\right) \frac{\partial B}{\partial T}\left(v, T_{n}\right),
$$

and then Eqs. (2.8), (2.9), and (2.17) determine $I_{n+1}$ and $T_{n+1}$. A bad feature of this approximation is that it is not positive for all $v$ if $T_{n+1}<T_{n}$. However, a good feature is that the error in Eq. (2.17) is $O\left(\Delta T^{2}\right)$. We call this method "linear" because the correction to $B_{n}$ in Eq. (2.17) is additive.

The "nonlinear" and "linear" methods can be rewritten as follows. Let us define

$$
\begin{gathered}
\tau_{n}=\frac{1}{c \Delta t_{n}}, \\
\Gamma_{n}(\mathbf{x}, v)= \begin{cases}\frac{4 B\left(v, T_{n}(\mathbf{x})\right)}{T_{n}(\mathbf{x})} & \text { (nonlinear) } \\
\frac{\partial B}{\partial T}\left(v, T_{n}(\mathbf{x})\right) & \text { (linear), }\end{cases} \\
\eta_{n}(\mathbf{x})=\frac{4 \pi \Delta t_{n} \int \sigma_{n} \Gamma_{n} d v^{\prime}}{c_{v, n}+4 \pi \Delta t_{n} \int \sigma_{n} \Gamma_{n} d v^{\prime}}, \\
\chi_{n}(\mathbf{x}, v)=\frac{\sigma_{n} \Gamma_{n}}{\int \sigma_{n} \Gamma_{n} d v^{\prime}},
\end{gathered}
$$

and

$$
Q_{n}(\mathbf{x}, \boldsymbol{\Omega}, v)=\tau_{n} I_{n}+\sigma_{n} B_{n}-\eta_{n} \chi_{n} \int \sigma_{n} B_{n} d v^{\prime}
$$

Then, $I_{n+1}$ and $T_{n+1}$ are determined by the equations

$$
\boldsymbol{\Omega} \cdot \nabla I_{n+1}+\left(\sigma_{n}+\tau_{n}\right) I_{n+1}=\eta_{n} \chi_{n} \frac{1}{4 \pi} \iint \sigma_{n} I_{n+1} d \Omega^{\prime} d \nu^{\prime}+Q_{n}
$$


and

$$
T_{n+1}=T_{n}+\frac{\Delta t_{n}}{c_{v, n}}\left(1-\eta_{n}\right) \iint \sigma_{n}\left(I_{n+1}-B_{n}\right) d \Omega^{\prime} d v^{\prime}
$$

Thus, the nonlinear radiative transfer problem has been reduced to solving Eq. (2.23) within each time step. This equation has the form of a steady-state linear neutron transport equation, with fission but no scattering [6,7]. Nevertheless, the integral term on the right side of this equation has been called the "pseudoscattering" term [1]. We note that for both the nonlinear and linear methods,

$$
\begin{gathered}
\Gamma_{n}>0, \\
0<\eta_{n}<1,
\end{gathered}
$$

and

$$
\int \chi_{n} d v^{\prime}=1
$$

\section{The LambDa Iteration Method}

The standard lambda iteration method for Eq. (2.23) is defined by

$$
\begin{gathered}
\mathbf{\Omega} \cdot \nabla I^{(k+1 / 2)}+(\sigma+\tau) I^{(k+1 / 2)}=\eta \chi F^{(k)}+Q, \\
F^{(k+1 / 2)}=\frac{1}{4 \pi} \iint \sigma I^{(k+1 / 2)} d \Omega^{\prime} d v^{\prime}, \\
F^{(k+1)}=F^{(k+1 / 2)},
\end{gathered}
$$

where each $I^{(k+1 / 2)}$ satisfies the boundary condition (2.4). We note that the timestep subscripts have been deleted, in recognition that $I$ and $F$ are unknown and that $\sigma, \tau, \eta, \chi$, and $Q$ are known, and that iteration superscripts have been added to the unknowns $I$ and $F$.

To analyze the convergence properties of the lambda method, let us define

$$
\begin{gathered}
\tilde{F}^{(k)}=F-F^{(k)}, \\
\tilde{I}^{(k+1 / 2)}=I-I^{(k+1 / 2),}
\end{gathered}
$$

where $F$ and $I$ satisfy Eqs. (3.1) and (3.2) with the iteration superscripts deleted (i.e., $I$ satisfies the desired transport equation). Then, the rate at which $\widetilde{F}^{(k)}$ and $\widetilde{I}^{(k+1 / 2)}$ tend to zero is identical to the rate at which $F^{(k)}$ and $I^{(k+1 / 2)}$ converge to $F$ and $I$. 
To determine this rate, we subtract Eqs. (3.1) through (3.3) from the equations for $F$ and $I$ to obtain the following equations for $\widetilde{F}^{(k)}$ and $\widetilde{I}^{(k+1 / 2)}$ :

$$
\begin{gathered}
\boldsymbol{\Omega} \cdot \nabla \tilde{I}^{(k+1 / 2)}+(\sigma+\tau) \tilde{I}^{(k+1 / 2)}=\eta \chi \widetilde{F}^{(k)}, \\
\tilde{F}^{(k+1)}=\frac{1}{4 \pi} \iint \sigma \widetilde{I}^{(k+1 / 2)} d \Omega^{\prime} d \nu^{\prime} .
\end{gathered}
$$

Now we take $\sigma, \eta$, and $\chi$ to be independent of $\mathbf{x}$ and consider a Fourier decomposition of $F^{(k)}$ and $I^{(k+1 / 2)}$. A single Fourier mode will then have the form

$$
\begin{aligned}
\widetilde{F}^{(k)} & =\omega^{k} e^{i \lambda \tilde{\xi} \cdot \mathbf{x},} \\
\widetilde{I}^{(k+1 / 2)} & =\omega^{k} a(\mathbf{\Omega}, v) e^{i \lambda \zeta \cdot x},
\end{aligned}
$$

where $|\xi|=1$ and $0<\lambda<\infty$. The Fourier mode with the largest value of $|\omega|$ will be the slowest to decay to zero if $|\omega|<1$, or it will be the most rapidly divergent if $|\omega|>1$. In either case, this mode determines the convergence properties of the iteration method, and we now seek to explicitly evaluate it.

Introducing Eqs. (3.8) and (3.9) into (3.6) and (3.7), we obtain

$$
\begin{gathered}
(i \lambda \xi \cdot \boldsymbol{\Omega}+\sigma+\tau) a=\eta \chi, \\
\omega=\frac{1}{4 \pi} \iint \sigma a d \Omega^{\prime} d v^{\prime},
\end{gathered}
$$

and thus

$$
\begin{gathered}
a(\mathbf{\Omega}, v)=\frac{\eta \chi(v)}{i \lambda \xi \cdot \Omega+\sigma(v)+\tau}, \\
\omega=\frac{1}{2} \int_{0}^{\infty} \int_{-1}^{1} \frac{\eta \sigma\left(v^{\prime}\right) \chi\left(v^{\prime}\right)}{i \lambda \mu+\sigma\left(v^{\prime}\right)+\tau} d \mu d v^{\prime} \\
=\eta \int_{0}^{\infty} \sigma\left(v^{\prime}\right) \chi\left(v^{\prime}\right)\left[\frac{1}{\lambda} \tan ^{-1} \frac{\lambda}{\sigma\left(v^{\prime}\right)+\tau}\right] d v^{\prime} .
\end{gathered}
$$

For each $v^{\prime}$, the bracketed term in Eq. (3.13) is a monotonic decreasing function of $\lambda$, taking its maximum value at $\lambda=0$. Thus,

$$
\begin{aligned}
\rho_{L} & =\sup _{i}|\omega| \\
& =\left.\omega\right|_{\lambda=0} \\
& =\eta \int_{0}^{\infty} \frac{\sigma\left(\nu^{\prime}\right) \chi\left(v^{\prime}\right)}{\sigma\left(v^{\prime}\right)+\tau} d v^{\prime} .
\end{aligned}
$$


The quantity $\rho_{\mathrm{L}}$ is termed the spectral radius of the lambda iteration scheme. By the above analysis, it determines the convergence rate for $I^{(k+1 / 2)}$ in the sense

$$
\left\|I-I^{(k+1 / 2)}\right\| \approx A \rho_{\mathrm{L}}^{k},
$$

where $A$ is a constant, \|\| is a suitable norm, and $k \gg 1$. Because of Eqs. (2.18), (2.26), and (2.27), we have $0<\rho_{\mathrm{L}}<1$, so the lambda method converges. However, wc also havc $\rho_{L} \rightarrow 1$ as $\eta \rightarrow 1$ and $\tau / \sigma \rightarrow 0$. By Eqs. (2.20) and (2.18),

$$
\eta \approx 1 \quad \text { for } \quad \frac{\sigma \Delta t}{c_{v}} \gg 1
$$

and

$$
\frac{\tau}{\sigma} \approx 0 \quad \text { for } \quad c \sigma \Delta t \gg 1 .
$$

Therefore, if the product $\sigma \Delta t$ is "large," then $\rho_{\mathrm{L}} \approx 1$, and although the lambda method converges, it does so very slowly.

Unfortunately, the conditions described by Eqs. (3.16) and (3.17) are often satisfied in practical situations. Here the only direct way to reduce $\rho_{\mathrm{L}}$ is to reduce $\Delta t$, but to have a sufficient impact on $\rho_{\mathrm{L}}$, one must usually take $\Delta t$ so small that the gains achieved by reducing the number of iterations per time step are overwhelmed by the large increase in the number of time steps. Thus, there is a need for a modified iteration algorithm with superior convergence properties. This is the purpose of the GTA method, and the subject of the remainder of this article.

Before proceeding, we reiterate that the slow convergence of the lambda method is due to the $\lambda \approx 0$ Fourier modes, for which Eq. (3.12) gives

$$
a(\mathbf{\Omega}, v)=\eta \frac{\chi(v)}{\sigma(v)+\tau}-i \lambda \eta \xi \cdot \frac{\mathbf{\Omega} \chi(v)}{[\sigma(v)+\tau]^{2}}+O\left(\lambda^{2}\right) .
$$

Thus, the two "shape" functions that are associated with the most slowly converging Fourier modes are

$$
\begin{gathered}
\Psi_{0}(v)=\frac{\chi(v)}{\sigma(v)+\tau}, \\
\boldsymbol{\Psi}_{1}(\boldsymbol{\Omega}, v)=\boldsymbol{\Omega} \frac{\chi(v)}{[\sigma(v)+\tau]^{2}} \equiv \mathbf{\Omega} \Psi_{1}(v) .
\end{gathered}
$$

\section{The GTA MeTHOD}

In the GTA method we begin each iteration with a lambda "sweep" described by Eqs. (3.1) and (3.2). However, we replace Eq. (3.3) by a more complicated, but 
more advantageous set of equations, derived as follows. First we define the grey frequency-integrated intensity and source

$$
\begin{gathered}
\hat{I}^{(k+1 / 2)}(\mathbf{x}, \mathbf{\Omega})=\int I^{(k+1 / 2)}\left(\mathbf{x}, \mathbf{\Omega}, v^{\prime}\right) d v^{\prime}, \\
\hat{Q}(\mathbf{x}, \mathbf{\Omega})=\int Q\left(\mathbf{x}, \mathbf{\Omega}, v^{\prime}\right) d v^{\prime},
\end{gathered}
$$

and we integrate Eq. (3.1) over $v$ and rearrange, obtaining

$$
\begin{aligned}
\mathbf{\Omega} \cdot \nabla & \hat{I}^{(k+1 / 2)}+\hat{\sigma}_{\mathrm{T}} \hat{I}^{(k+1 / 2)}-\frac{\hat{\sigma}_{\mathrm{S}}}{4 \pi} \int \hat{I}^{(k+1 / 2)} d \Omega^{\prime} \\
= & \int\left(\hat{\sigma}_{\mathrm{T}}-\sigma-\tau\right) I^{(k+1 / 2)} d v^{\prime}+\eta F^{(k)}-\frac{\hat{\sigma}_{\mathrm{S}}}{4 \pi} \iint I^{(k+1 / 2)} d \Omega^{\prime} d v^{\prime}+\hat{Q} .
\end{aligned}
$$

In deriving this equation, we used the normalization (2.27) for $\chi$, and we added identical terms containing the (for now) undefined grey opacities $\hat{\sigma}_{\mathrm{T}}$ and $\hat{\sigma}_{\mathrm{S}}$ to both sides of Eq. (4.3). (These quantities are derived later and are not obtained by directly integrating $\sigma_{\mathrm{T}}$ or $\sigma_{\mathrm{S}}$ over $v$, as in Eqs. (4.1) and (4.2).) To define an equation for $\hat{I}^{(k+1)}$, we replace $F^{(k)}$ by $F^{(k+1 / 2)}$ on the right side of Eq. (4.3); using Eq. (3.2), we obtain

$$
\begin{aligned}
\mathbf{\Omega} \cdot \nabla & \hat{I}^{(k+1)}+\hat{\sigma}_{\mathrm{T}} \hat{I}^{(k+1)}-\frac{\hat{\sigma}_{\mathrm{S}}}{4 \pi} \int \hat{I}^{(k+1)} d \Omega^{\prime} \\
\quad & =\left[\int\left(\hat{\sigma}_{\mathrm{T}}-\sigma-\tau\right) I^{(k+1 / 2)} d v^{\prime}+\frac{1}{4 \pi} \iint\left(\eta \sigma-\hat{\sigma}_{\mathrm{S}}\right) I^{(k+1 / 2)} d \Omega^{\prime} d v^{\prime}\right]+\hat{Q},
\end{aligned}
$$

where $\hat{I}^{(k+1)}$ satisfies the same incident boundary conditions as $\hat{I}^{(k+1 / 2)}$. We note that Eqs. (4.3) and (4.4) become identical upon convergence. Next, to obtain $F^{(k+1)}$, we rewrite Eq. (3.2) as

$$
F^{(k+1 / 2)}=\frac{\hat{\sigma}}{4 \pi} \int \hat{I}^{(k+1 / 2)} d \Omega^{\prime}+\frac{1}{4 \pi} \iint(\sigma-\hat{\sigma}) I^{(k+1 / 2)} d \Omega^{\prime} d v^{\prime},
$$

where the grey opacity $\hat{\sigma}$ is (for now) undetermined, and then we define

$$
F^{(k+1)}=\frac{\hat{\sigma}}{4 \pi} \int \hat{I}^{(k+1)} d \Omega^{\prime}+\left[\frac{1}{4 \pi} \iint(\sigma-\hat{\sigma}) I^{(k+1 / 2)} d \Omega^{\prime} d v^{\prime}\right] .
$$

We note that Eqs. (4.5) and (4.6) become identical upon convergence.

The GTA method is given by Eqs. (3.1), (4.4), and (4.6). Equivalently, if we define

$$
\psi^{(k+1)}(\mathbf{x}, \mathbf{\Omega})=\hat{I}^{(k+1)}(\mathbf{x}, \mathbf{\Omega})-\hat{I}^{(k+1 / 2)}(\mathbf{x}, \mathbf{\Omega})
$$


then, by subtracting Eq. (4.4) from (4.3) and (4.6) from (4.5) and using Eq. (3.2), we obtain

$$
\begin{gathered}
\mathbf{\Omega} \cdot \nabla I^{(k+1 / 2)}+(\sigma+\tau) I^{(k+1 / 2)}=\eta \chi F^{(k)}+Q, \\
F^{(k+1 / 2)}=\frac{1}{4 \pi} \iint \sigma I^{(k+1 / 2)} d \Omega^{\prime} d v^{\prime}, \\
\mathbf{\Omega} \cdot \nabla \psi^{(k+1)}+\hat{\sigma}_{\mathrm{T}} \psi^{(k+1)}-\frac{\hat{\sigma}_{\mathrm{S}}}{4 \pi} \int \psi^{(k+1)} d \Omega^{\prime}=\eta\left[F^{(k+1 / 2)}-F^{(k)}\right], \\
F^{(k+1)}=F^{(k+1 / 2)}+\frac{\hat{\sigma}}{4 \pi} \int \psi^{(k+1)} d \Omega^{\prime} .
\end{gathered}
$$

In this form, the grey equation (4.10) has a much simpler source term than in Eq. (4.4). Also, we have taken the incident flux for $I$ on the exterior boundary to be prescribed (see Eq. (2.4)), and thus, since $\hat{I}^{(k+1 / 2)}$ and $\hat{I}^{(k+1)}$ have the same prescribed incident boundary values,

$$
\psi^{(k+1)}(\mathbf{x}, \mathbf{\Omega})=0, \quad \mathbf{x} \in \partial D, \quad \mathbf{\Omega} \cdot \mathbf{n}<0 .
$$

(If part of the boundary conditions for $I$ are reflecting, then this same part will also be reflecting for $\psi^{(k+1)}$.) Equation (4.12) is now a simpler boundary condition than the one which holds for $\hat{I}^{(k+1)}$, and $\psi^{(k+1)}$ is fully specified by Eqs. (4.10) and (4.12).

To summarize, the GTA method is described by Eqs. (4.8)-(4.12). The first two of these equations are identical to the first two steps in the lambda method (Eqs. (3.1) and (3.2)), but the remaining three GTA equations are fundamentally different from the last equation in the lambda method (Eq. (3.3)).

In particular, these remaining GTA equations contain the grey transport equation (4.10). Therefore, in one "outer" iteration, consisting of a single pass through Eqs. (4.8)-(4.12), one is required to solve Eq. (4.10), and to do this one must typically perform "inner" iterations. (Unless stated otherwise, we assume in this paper that the grey equation (4.10) is solved by lambda iteration. Other possible iteration strategies are discussed in Secton VIII.) Thus, the GTA method is, like DSA, a nested algorithm that has two spectral radii associated with it, one $\left(\rho_{\text {OUT }}\right)$ corresponding to the reduction of the iteration error in $F$ during an outer iteration, and the other $\left(\rho_{\mathrm{IN}}\right)$ corresponding to the reduction of the iteration error in $\psi$ during an inner iteration. The Fourier analysis for the outer and inner iterations can be performed just as described in Secton III. For outer iterations, we obtain a complicated expression that must be evaluated numerically (see Section VI), but for inner iteratons we easily obtain

$$
\rho_{\mathrm{IN}}=\left|\frac{\hat{\sigma}_{\mathrm{S}}}{\hat{\sigma}_{\mathrm{T}}}\right| \text {. }
$$


To fully specify the GTA method, it remains to determine the grey opacities $\hat{\sigma}_{I}$, $\hat{\sigma}_{\mathrm{s}}$, and $\hat{\sigma}$; we do this in the next section.

\section{The Grey Opacities}

In Secton III we showed that the most slowly converging components of $I$ in the lambda iteration method are $\Psi_{0}$ and $\Psi_{1}$ (Eqs. (3.19) and (3.20)), and in Section $I V$ we derived a generalization of the lambda method (Eqs. (4.8) (4.12)) containing three free parameters - the grey opacities $\hat{\sigma}_{\mathrm{T}}, \hat{\sigma}_{\mathrm{S}}$, and $\hat{\sigma}$. In this section we define these grey opacities by requiring the GTA algorithm to converge in one iteration if $I^{(k+1 / 2)}$ is a linear combination of $\Psi_{0}(v)$ and $\xi(\mathbf{x}) \cdot \Psi_{1}(\Omega, v)$, for an arbitrary vector function $\xi(\mathbf{x})$.

To do this, it is convenient to consider he GTA method as defined by Eqs. (3.1), (3.2), (4.4), and (4.6). Let us require the bracketed terms on the right sides of Eqs. (4.4) and (4.6) to vanish if $I^{(k+1 / 2)}$ has either the shape of $\Psi_{0}$ or $\xi$. These conditions can be shown to guarantee $\omega(0)=0$, and they yield the three equations

$$
\begin{aligned}
& 0=\int\left(\hat{\sigma}_{\mathrm{T}}-\sigma-\tau+\eta \sigma-\hat{\sigma}_{\mathrm{S}}\right) \Psi_{0} d v^{\prime} \\
& 0=\int\left(\hat{\sigma}_{\mathrm{T}}-\sigma-\tau\right) \Psi_{1} d v^{\prime} \\
& 0=\int(\sigma-\hat{\sigma}) \Psi_{0} d v^{\prime}
\end{aligned}
$$

which have the solutions

$$
\begin{aligned}
\hat{\sigma} & =\int \frac{\sigma \chi}{\sigma+\tau} d v^{\prime} / \int \frac{\chi}{\sigma+\tau} d v^{\prime}, \\
\hat{\sigma}_{\mathrm{T}} & =\int \frac{\chi}{\sigma+\tau} d v^{\prime} / \int \frac{\chi}{(\sigma+\tau)^{2}} d v^{\prime}, \\
\hat{\sigma}_{\mathrm{S}} & =\hat{\sigma}_{\mathrm{T}}-(1-\eta) \hat{\sigma}-\tau .
\end{aligned}
$$

Equations (5.2) have the following properties:

(i) $0<\hat{\sigma}_{\mathrm{T}}$ and $\hat{\sigma}_{\mathrm{S}}<\hat{\sigma}_{\mathrm{T}}$. Thus, if $0 \leqslant \hat{\sigma}_{\mathrm{S}}$, then the grey equation (4.10) can be solved by lambda iteration. However, if $\hat{\sigma}_{\mathrm{S}}<0$, then one can show that Eqs. $(5.1 \mathrm{a})$ and $(5.1 \mathrm{c})$, which imply $(5.2 \mathrm{c})$ and $(5.2 \mathrm{a})$, give $\omega(0)=0$. Thus, we can abandon Eq. $(5.2 \mathrm{~b})$, set $\hat{\sigma}_{\mathrm{S}}=0$, and use Eq. $(5.2 \mathrm{c})$ to give $\hat{\sigma}_{\mathrm{T}}=(1-\eta) \delta+\tau$. Then we still have $\omega(0)=0$, and because $\hat{\sigma}_{\mathrm{S}}=0$, the lambda iteration scheme converges with just one inner iteration per outer. These "default" values of $\hat{\sigma}_{T}$ and $\hat{\sigma}_{S}$ are used only when $\sigma \Delta t$ is sufficiently small, i.e., for situations when lambda iteration itself is reasonably quickly converging. 
(ii) Letting $\rho_{\mathbf{L}}$ denote the spectral radius of the lambda iteration method applied to the multifrequency equation (2.23) (see Eq. (3.14)), and $\rho_{\text {IN }}$ denote the spectral radius of the lambda iteration method applied to the grey equation (4.10) (see Eq. (4.13)), we have

$$
\rho_{\mathrm{IN}}=\frac{\hat{\sigma}_{\mathrm{S}}}{\hat{\sigma}_{\mathrm{T}}}=1-\frac{\zeta_{2}}{\zeta_{1}^{2}}(1-\eta) \int \frac{\sigma \chi}{\sigma+\tau} d v^{\prime}-\frac{\zeta_{2}}{\zeta_{1}} \tau,
$$

where

$$
\zeta_{n}=\int \frac{\chi}{(\sigma+\tau)^{n}} d v^{\prime}, \quad n=1,2
$$

After some algebra, we obtain

$$
\rho_{\mathrm{L}}=\rho_{\mathrm{IN}}+\left(\frac{\zeta_{2}}{\zeta_{1}^{2}}-1\right)\left(1-\eta+\eta \tau \chi_{1}\right)
$$

where, by Cauchy's inequality and Eq. (2.27),

$$
\zeta_{1}^{2}=\left[\int \chi^{1 / 2} \frac{\chi^{1 / 2}}{\sigma+\tau} d v^{\prime}\right]^{2} \leqslant \int \frac{\chi}{(\sigma+\tau)^{2}} d v^{\prime}=\zeta_{2} .
$$

Therefore,

$$
0 \leqslant \rho_{I N} \leqslant \rho_{L},
$$

with the right equality holding iff $\Delta t=\infty$ or $\sigma(v)=$ constant, and the left equality holding whenever the formulas (5.2) lead to $\hat{\sigma}_{\mathrm{S}}<0$. Hence, for $\Delta t<\infty$ and $\sigma(v) \neq$ constant, the grey equation (4.10) can be solved by lambda iteration more efficiently than the original multifrequency equation (2.23).

(iii) If $\sigma(v)=$ constant, then $\rho_{\mathrm{IN}}=\rho_{\mathrm{L}}$, but

$$
\begin{aligned}
\hat{\sigma} & =\sigma, \\
\hat{\sigma}_{\mathrm{T}} & =\sigma+\tau, \\
\hat{\sigma}_{\mathrm{S}} & =\eta \sigma,
\end{aligned}
$$

and from Eqs. (4.4) and (4.6), the GTA method will converge in one iteration.

\section{Fourier ANALysis}

Here we describe the results of Fourier analyses that predict values of $\rho_{\text {out }}$ and $\rho_{\text {IN }}$ for idealized infinite-medium problems in which $\gamma, c_{v}$, and $T$ are independent of $\mathbf{x}$. These analyses use the forms (2.6) and (2.7) for $c_{v}$ and $\sigma$, and are based on the nonlinear semi-implicit time differencing method described in Section II. (The convergence rates for the linear semi-implicit time differencing are slightly faster.) 
To numerically solve the radiative transfer equations, it is necessary to discretize $\mathbf{x}, \boldsymbol{\Omega}$, and $v$. Typically, $\mathbf{x}$ is discretized by a finite difference or finite element method, $\boldsymbol{\Omega}$ by a finite element or discrete-ordinates approximation, and $v$ by the multigroup method [2-8]. We observe in computations that the $\mathbf{x}$ and $v$ discretizations do not appreciably affect the inner or outer iteration convergence rates, and that the choice of the $\boldsymbol{\Omega}$-discretization does affect the outer convergence rate but not the inner. Therefore, we have considered the slab-geometry discrete-ordinate versions of Eqs. (4.8)-(4.12) and have evaluated $\rho_{\text {OUT }}$ for the worst case of $\Delta t=\infty$, and for the standard Gauss-Legendre angular quadrature $[6,7]$ sets of various orders $N Q$. The Fourier analysis for this algorithm is implemented just as described in Section III, with $c_{v}, \gamma$, and $T$ taken to be constants. The resulting expression for $\omega_{\text {out }}(\lambda)$, after a considerable amount of algebraic manipulation using Eqs. (2.5), (2.7), (2.19), and (2.21), is

$$
\omega_{\mathrm{OUT}}(\lambda)=g(\Lambda) \frac{f(\Lambda)}{v_{2}} \frac{1-g(\Lambda)}{1-f(\Lambda)},
$$

where

$$
\begin{gathered}
v_{n}=\frac{\left[\int_{0}^{\infty} e^{-t}\left(t^{3} /\left(1-e^{-t}\right)\right) d t\right]^{n}}{\int_{0}^{\infty} e^{-t}\left(t^{3} /\left(1-e^{-t}\right)\right)^{2} d t}, \quad n=1,2, \\
\Lambda=\frac{\lambda}{\hat{\sigma}_{\mathrm{T}}}, \\
f(\Lambda)=\sum_{\mu_{m}>0} \frac{1}{1+\left(\Lambda \mu_{m}\right)^{2}} w_{m}, \\
g(\Lambda)=\sum_{\mu_{m}>0} \int_{0}^{\infty}\left(e^{-t} /\left(1+\left[v_{1} \frac{t^{3}}{1-e^{-t}} \lambda \mu_{m}\right]^{2}\right)\right) d t W_{m}
\end{gathered}
$$

Here $\mu_{m}$ are the discrete ordinate values, and $w_{m}$ are the angular weights $[2,6,7]$, satisfying

$$
\sum_{\mu_{m}>0} w_{m}=1
$$

By expanding the above expressions for $|\Lambda| \ll 1$, one can easily show $\omega_{\text {Out }}(0)=0$. Also, these equations are independent of $c_{v}$, and variations in $\gamma$ and $T$ have the sole effect of rescaling $\lambda$ in Eq. (6.3). Therefore, $\rho_{\text {OUT }}$ is independent of $c_{v}, \gamma$, and $T$. (This is true only for the special case of $\Delta t=\infty$ considered above.)

In addition to evaluating $\rho_{\text {our }}$, we have also computed $N_{\text {OUT }}$, the number of outer iterations necessary to reduce the iteraton error by one order of magnitude:

$$
\left(\rho_{\text {OUT }}\right)^{N_{\text {OUT }}}=0.1 \text {. }
$$

The results of our calculations are summarized in Table $\mathrm{I}$. 
TABLE I

$\rho_{\text {OUT }}$ and $N_{\text {OUT }}$ for Various Values of $N Q$

\begin{tabular}{rcc}
\hline$N Q$ & $\rho_{\text {OUT }}$ & $N_{\text {OUT }}$ \\
\hline 2 & 0.867 & 16.1 \\
4 & 0.844 & 13.6 \\
8 & 0.810 & 10.9 \\
16 & 0.770 & 8.8 \\
$\infty$ & 0.706 & 6.6 \\
\hline
\end{tabular}

We see that $\rho_{\text {OUT }}$ is a decreasing function of $N Q$, taking its smallest value for the hypothetical case of an infinite-order quadrature set, corresponding to no angular discretization. We emphasize that for this worst case of $\Delta t=\infty$, we have $\rho_{\mathrm{I}}=1.0$ and $N_{\mathrm{L}}=\infty$, and thus the lambda method for the multigroup equations can, in practical problems, converge arbitrarily slowly. However, this is not true for the GTA method, as is shown in Table I.

Regarding inner iterations, we have by Eqs. (3.14) and (5.3), for the worst case $\Delta t=\infty$,

$$
\rho_{\mathrm{IN}}=\rho_{\mathrm{L}}=1.0
$$

However, for $\Delta t<\infty$, we have by Eqs. (3.14) and (5.7),

$$
\rho_{\mathrm{IN}}<\rho_{\mathrm{L}}<1.0
$$

To illustrate the relative values of $\rho_{\mathrm{IN}}$ and $\rho_{\mathrm{L}}$ for $\Delta t<\infty$, we have evaluated $\rho_{\mathrm{IN}}$, $N_{\text {IN }}, \rho_{\mathrm{L}}$, and $N_{\mathrm{L}}$ for various values of $\eta$ and $\tau$, with the model opacity given by Eq. (2.7), and the numerical values of the constants $c_{v}$ and $\gamma$ in Eqs. (2.6) and (2.7) set equal to one; the results are given in Table II. Here we see that in the worst cases, where $N_{\mathrm{IN}}$ and $N_{\mathrm{L}}$ are large, $N_{\mathrm{L}}$ is larger than $N_{\mathrm{IN}}$ by about a factor of 18 . Therefore, the grey equation (4.10) will require less work to be solved by lambda iteration than the multifrequency equation (2.23) for two reasons: Eq. (4.10) has a smaller spectral radius, and only one frequency group. We emphasize that the worst case result $N_{\mathrm{L}} / N_{\text {IN }} \approx 18$ applies to the model opacities given by Eq. (2.7), and that this ratio can change for other opacities.

To implement the Fourier analysis, we have made the idealized assumptions that the medium is infinite and that $\gamma, c_{v}$, and $T$ are constants. In the next section we present experimental results from numerical solutions of the radiative transfer cquations using the GTA algorithm. These results show that convergence rates predicted by the Fourier analysis represent approximate upper bounds for problems in finite media, with variable $\gamma, c_{v}$, and $T$, and a nonuniform spatial mesh. 
TABLE II

The Quantities $\rho_{\mathrm{L}}\left(N_{\mathrm{L}}\right)$ and $\rho_{\mathrm{IN}}\left(N_{\mathrm{IN}}\right)$ for Various Values of $\eta$ and $\tau$

\begin{tabular}{|c|c|c|c|c|c|c|}
\hline & \multicolumn{2}{|c|}{0.0} & \multicolumn{2}{|c|}{0.001} & \multicolumn{2}{|c|}{0.01} \\
\hline 1.0 & $\begin{array}{l}1.0 \\
1.0\end{array}$ & $\begin{array}{l}(\infty) \\
(\infty)\end{array}$ & $\begin{array}{l}0.994 \\
0.929\end{array}$ & $\begin{array}{l}(382.6) \\
(31.3)\end{array}$ & $\begin{array}{l}0.958 \\
0.750\end{array}$ & $\begin{array}{c}(53.6) \\
(8.0)\end{array}$ \\
\hline 0.999 & $\begin{array}{l}0.999 \\
0.983\end{array}$ & $\begin{array}{c}(2301.8) \\
(134.3)\end{array}$ & $\begin{array}{l}0.993 \\
0.917\end{array}$ & $\begin{array}{c}(327.8) \\
(26.6)\end{array}$ & $\begin{array}{l}0.957 \\
0.744\end{array}$ & $\begin{array}{c}(52.4) \\
(7.8)\end{array}$ \\
\hline 0.99 & $\begin{array}{l}0.990 \\
0.826\end{array}$ & $\begin{array}{c}(229.1) \\
(12.0)\end{array}$ & $\begin{array}{l}0.984 \\
0.810\end{array}$ & $\begin{array}{c}(142.8) \\
(10.9)\end{array}$ & $\begin{array}{l}0.948 \\
0.693\end{array}$ & $\begin{array}{c}(43.1) \\
(6.3)\end{array}$ \\
\hline
\end{tabular}

\section{NUMERICAL RESULTS}

In this section we discuss convergence of a numerical solution of the azimuthally symmetric, slab geometry problem

$$
\begin{gathered}
\frac{1}{c} \frac{\partial I}{\partial t}+\mu \frac{\partial I}{\partial x}=\sigma(B-1), \\
c_{v} \frac{\partial T}{\partial t}=2 \pi \iint \sigma(I-B) d \mu^{\prime} d v^{\prime},
\end{gathered}
$$

with the initial conditions

$$
\begin{gathered}
I(x, v, \mu, 0)=B\left(v, T_{i}\right), \quad 0<x<X, \\
T(x, 0)=T_{i},
\end{gathered}
$$

the boundary conditions

$$
\begin{array}{ll}
I(0, \mu, v, t)=B\left(v, T_{b}\right), & 0<t, \quad 0<\mu<1, \\
I(X, \mu, v, t)=0, & 0<t,-1 \leqslant \mu<0,
\end{array}
$$

and the opacity defined by

$$
\sigma(x, v, T)=\gamma(x) \frac{1-e^{-h v / k T}}{(h v)^{3}} .
$$


Here $x$ is measured in centimeters, $t$ in seconds, $v$ in seconds ${ }^{-1}$, and $T$ in degrees Kelvin, and we take

$$
\begin{aligned}
X & =4 \mathrm{~cm}, \\
\frac{c_{v}}{k} & =5.109 \times 10^{14} \mathrm{~cm}^{-3}, \\
k T_{i} & =1.0 \mathrm{eV}, \\
k T_{b} & =1.0 \mathrm{keV}, \\
\gamma(x) & =\left\{\begin{array}{lll}
1 & \mathrm{keV}^{3} / \mathrm{cm} & 0<x<1, \\
1000 \mathrm{keV}^{3} / \mathrm{cm} & 1<x<2, \\
1 & \mathrm{keV}^{3} / \mathrm{cm} & 2<x<4 .
\end{array}\right.
\end{aligned}
$$

This fully specifies the physical problem.

We discretize this problem as follows. The time variable is treated using the linear semi-implicit time differencing method described in Section II, with $\Delta t=3 \times 10^{-11} \mathrm{~s}$. The angular variable $\mu$ is treated using the standard discrete-ordinates method $[2,6,7]$ with the $S_{4}$ Gauss-Legendre quadrature set. The frequency variable $v$ is treated by the multigroup approximation, with 50 groups logarithmically spaced between $h v_{\max }=10 \mathrm{keV}$ and $h v_{\min }=10^{-5} \mathrm{keV}$. Thus, the $g$ th group is defined by

$$
v_{g+1 / 2}<v<v_{g-1 / 2}
$$

with

$$
v_{g+1 / 2}=v_{\min }^{g / 50} v_{\max }^{1-g / 50}, \quad 0 \leqslant g \leqslant 50 .
$$

The spatial mesh is nonuniform and defined by

$$
\Delta x= \begin{cases}0.1 \mathrm{~cm} & 0<x<1 \\ 0.02 \mathrm{~cm} & 1<x<2 \\ 0.2 \mathrm{~cm} & 2<x<4\end{cases}
$$

Thus, there are a total of 70 spatial cells. Within each cell, the material temperature $T$ is taken to be constant, and Eqs. (4.8) and (4.10) are both discretized by the standard linear discontinuous method $[7,12]$.

The problem is now fully discretized. At the beginning of each time step the material temperatures $T$ are known in each cell, and thus the multigroup opacity, given by Eq. (7.6), and the grey opacities, given by Eqs. (5.2), are evaluated for each individual cell.

To implement the GTA method, one is theoretically supposed to perform inner iterations to fully converge the grey equation (4.10) within each outer iteration. We have experimentally found that it is not necessary to do this. However, in reducing the number of inner iterations too much, one slows the convergence of the outer 
iteration process, and then more outer iterations are reqired. Because multigroup transport sweeps are much more expensive than grey transort sweeps, the overall efficiency is thereby reduced. Hence, it is generally necessary to be sure that the grey equation is sufficiently converged within each time step. Operationally, we have found that the best way to insure this is to fix the number $\left(N_{\mathrm{IO}}\right)$ of inner iterations per outer iteration. The optimal value of $N_{\mathrm{IO}}$ is problem-dependent, and as might be expected, it increases as $\rho_{\mathrm{IN}}$ increases. For the problem considered here, we selected $N_{\text {IO }}=20$.

To determine outer iteration errors and convergence rates, we define

$$
\begin{aligned}
F_{j}^{(k+1 / 2)} & =\frac{1}{2} \sum_{g=1}^{50} \sum_{m=1}^{4} \sigma_{g j} I_{g m j}^{(k+1 / 2)} w_{m} \Delta v_{g}, \\
E^{(k)} & =\left\{\frac{1}{4} \sum_{j=1}^{70}\left[F_{j}^{(k+1 / 2)}-F_{j}^{(k-1 / 2)}\right]^{2} \Delta x_{j}\right\}^{1 / 2},
\end{aligned}
$$

where $I_{g m j}^{(k+1 / 2)}$ is the cell-average intensity for the $g$ th frequency group, at angle $\mu_{m}$, in the $j$ th spatial cell. We stop iterating when $E^{(k)}<10^{-4}$. Estimates of the spectral radius are then made by monitoring the quantity

$$
R^{(k)}=E^{(k)} / E^{(k-1)} .
$$

A similar procedure is used to monitor the spectral radius for inner iterations.

In Table III we summarize our observed results. Here the theoretical values of $\rho_{\mathrm{I}}$ and $\rho_{\text {IN }}$ are computed by evaluating the expressions (3.14) and (4.13) in each cell and taking the maximum value over the 70 cells, and the theoretical value of $\rho_{\text {out }}$ was taken from Table I. The observed values of $\rho_{\mathrm{L}}$ are sometimes larger than the theoretical values, but only by a very small amount. The observed values of $\rho_{\mathrm{IN}}$ are all close to and less than or equal to the theoretical values, and the observed values of $\rho_{\text {OUT }}$ are all less than the theoretical values, sometimes by a substantial amount.

A detailed description of the convergence of the outer iterations is given in Table IV, where $R^{(k)}$ is tabulated for the first four time steps. The results for greater

\begin{tabular}{|c|c|c|c|c|c|c|}
\hline \multirow[b]{2}{*}{ Time step } & \multicolumn{2}{|c|}{$\rho_{\mathbf{L}}$} & \multicolumn{2}{|c|}{$\rho_{\mathrm{IN}}$} & \multicolumn{2}{|c|}{ Pout } \\
\hline & Theoretical & Observed & Theoretical & Observed & Theoretica! & Observed \\
\hline 1 & 0.9838 & 0.9837 & 0.7995 & 0.7995 & 0.844 & 0.011 \\
\hline 2 & 0.9839 & 0.9844 & 0.7996 & 0.7614 & 0.844 & 0031 \\
\hline 3 & 0.9839 & 0.9842 & 0.7997 & 0.7726 & 0.844 & 0.495 \\
\hline 4 & 0.9839 & 0.9837 & 0.7997 & 0.7820 & 0.844 & 0.632 \\
\hline
\end{tabular}

TABLE III

Theoretical and Observed Spectral Radii 
TABLE IV

$R(k)$ for Time Sleps One through Four

\begin{tabular}{r|cccc}
\hline & Time step & 1 & 3 & \\
\hline & & 2 & & \\
\hline 2 & 0.0111 & 0.0559 & 0.4973 & 0.3294 \\
3 & 0.0112 & 0.0387 & 0.5480 & 0.5830 \\
4 & 0.0112 & 0.0311 & 0.4667 & 0.5578 \\
5 & & & 0.4542 & 0.5829 \\
6 & & 0.4571 & 0.6063 \\
7 & & 0.4641 & 0.6229 \\
8 & & 0.4718 & 0.6323 \\
9 & & 0.4789 & 0.6367 \\
10 & & 0.4848 & 0.6380 \\
11 & & 0.4893 & 0.6377 \\
12 & & 0.4926 & 0.6367 \\
13 & & 0.4947 & 0.6356 \\
14 & & & 0.6344 \\
15 & & & 0.6334 \\
16 & & & 0.6326 \\
17 & & & & 0.6319 \\
\hline
\end{tabular}

time steps are essentially the same as for time step 4 . Thus, since time step 4 is typical, we shall discuss it in detail.

Each GTA outer iteration consists of one multigroup transport sweep (for 50 groups) followed by 20 one-group transport sweeps; this is equivalent to 1.4 multigroup sweeps. Multiplying this by 17, we find that, effectively, 23.8 multigroup transport sweeps are performed in this time step. In the lambda iteration method, 850 multigroup transport sweeps are required; thus, the GTA algorithm represents a speedup factor of about 36 . The cost of the overhead needed to generate the grey opacities is very low and does not appreciably affect the speedup factor.

We have tested the GTA method for a large variety of problems, with other spatial and time-differencing schemes $[13,14]$, and the speedups reported above are actually less than those observed in problems with larger values of $\sigma$ or $\Delta t$, where we typically see speedups in the range of 50-80.

For smaller $\sigma$ or $\Delta t$, we observe smaller speedup factors. In particular, if $\Delta t$ is sufficiently small that the "default" expressions for $\hat{\sigma}_{\mathrm{T}}$ and $\hat{\sigma}_{\mathrm{S}}$ are used, then speedup factors of about 3.0 occur. (However, we reiterate that in such problems, lambda iteration itself converges fairly rapidly.) Also, if a spatial differencing scheme with a ncgative flux fixup [7] is used, then in problems containing too many negative flux fixups, a degradation of the convergence rate or even an instability (lack of convergence) can occur [13]. 
A crude formula for the speedup factor can be derived for large $\sigma \Delta t$, in which case $\rho_{\mathrm{L}}$ and $\rho_{\mathrm{IN}}$ are very close to one. Here we have

$$
N_{\mathrm{L}}=\frac{\ln 0.1}{\ln \rho_{\mathrm{L}}}=\frac{\ln 0.1}{\ln \left[1-\left(1-\rho_{\mathrm{L}}\right)\right]} \approx \frac{\ln 10}{1-\rho_{\mathrm{L}}},
$$

and, likewise,

$$
N_{\mathrm{IN}} \approx \frac{\ln 10}{1-\rho_{\mathrm{IN}}}
$$

Therefore,

$$
N_{\mathrm{IN}} \approx \frac{1-\rho_{\mathrm{L}}}{1-\rho_{\mathrm{IN}}} N_{\mathrm{L}} .
$$

Now, let $W_{\mathrm{L}}$ denote the work done in the lambda method to reduce the outer iteration error by one order of magnitude. If work is measured in units of the computational effort required to perform one grey transport sweep, then

$$
W_{\mathrm{L}}=N_{\mathrm{L}} N_{\mathrm{G}},
$$

where $N_{G}$ is the number of groups. Using the tyical value $\rho_{\text {OUT }} \approx 0.8$, for which $N_{\text {OUT }} \approx 10$, we find that

$$
W_{\mathrm{GTA}}=10\left(N_{\mathrm{G}}+N_{\mathrm{IN}}\right)
$$

Using Eqs. (7.16)-(7.18), we obtain for large $N_{\mathrm{L}}$ a speedup factor of

$$
\frac{W_{\mathrm{L}}}{W_{\mathrm{GTA}}} \approx \frac{1-\rho_{\mathrm{IN}}}{1-\rho_{\mathrm{L}}} \frac{N_{\mathrm{G}}}{10} \text {. }
$$

For the opacities used in this article, we have for $\rho_{\mathrm{IN}} \approx \rho_{\mathrm{L}} \approx 1$,

$$
\frac{1-\rho_{\mathrm{IN}}}{1-\rho_{\mathrm{L}}} \approx 18
$$

Therefore, with $N_{\mathrm{G}}=50$, we obtain

$$
\frac{W_{L}}{W_{\text {GTA }}} \approx 90
$$

This is an upper bound on the speedups that we observed in numerical calculations.

We note that as $\sigma \Delta t \rightarrow \infty, N_{\mathrm{L}}$ and $N_{\mathrm{IN}}$ both tend to $\infty$. For this situation, in the GTA algorithm onc will spend most of the time performing grey iterations. If this inner iteration process could by accelerated, it would greatly affect the speedup factor. This and other matters are discussed in the next section. 


\section{Discussion}

The GTA algorithm described in this article has the property that the terms that are not accelerated in the grey equation (4.10) are additive. It is also possible to formulate a GTA method in which the terms which are not accelerated are multiplicative. For example, if we integrate Eq. (3.1), over $v$ and use Eq. (4.1), we can obtain the acceleration equations

$$
\begin{gathered}
\mathbf{\Omega} \cdot \nabla \hat{I}^{(k+1)}+\left[\frac{\int(\sigma+\tau) I^{(k+1 / 2)} d v^{\prime}}{\int I^{(k+1 / 2)} d v^{\prime}}\right] \hat{I}^{(k+1)} \\
=\frac{1}{4 \pi}\left[\eta \frac{\iint \sigma I^{(k+1 / 2)} d \Omega^{\prime} d v^{\prime}}{\iint I^{(k+1 / 2)} d \Omega^{\prime} d v^{\prime}}\right] \int \hat{I}^{(k+1)} d \Omega^{\prime}+\int Q d v^{\prime}, \\
F^{(k+1)}=F^{(k+1 / 2)}\left[\frac{\int \hat{I}^{(k+1)} d \Omega^{\prime}}{\int \hat{I}^{(k+1 / 2)} d \Omega^{\prime}}\right] .
\end{gathered}
$$

These equations are the transport-theory analog of the nonlinear multifrequencygrey method that has been successfully employed in the diffusion approximation to the radiative transfer equations $[2,8]$. Equation $(8.1)$, however, has at least two shortcomings:

(1) There is no guarantee that for each time step, the effective total cross section [the bracketed term on the left side of Eq. (8.1)] dominates the effective scaltering cross section (the bracketed term on the right side of Eq. (3.1)). Thus, there is no guarantee that Eq. (8.1) possesses a solution for arbitrary $I^{(k+1 / 2)}$.

(2) The effective total cross section in Eq. (8.1) is dependent on $\boldsymbol{\Omega}$. This is nonstandard, and it may cause stability problems for iteration algorithms even if a solution of Eq. (8.1) exists.

Because of these difficulties, it seems unlikely that this nonlinear multifrequency grey approach will be either as effective or as robust as the linear GTA method.

Finally, we discuss some ways in which the GTA algorithm might be modified to become more efficient.

First, the GTA method is a nested algorithm requiring, in general, numerous inner iterations per outer iteration. Also, the GTA method appears to perform best when the number of inners per outer, $N_{\text {IO }}$, is preassigned and fixed within each outer iteration. For example, in the problem considered in Section VII, $N_{\text {IO }}$ satisfies, for each time step,

$$
\left(\rho_{\mathrm{IN}}\right)^{N_{\mathrm{IO}}} \leqslant 10^{-4},
$$

but it is not known whether this or another definition of $N_{\mathrm{IO}}$ is best. Further work on this will be required to optimize the GTA method.

Another possible way to improve the efficiency of the GTA method is to accelerate the lambda method for convergence of the inner iterations. In particular, 
as $\sigma \Delta t \rightarrow \infty$, the spectral radii $\rho_{\mathrm{L}}$ and $\rho_{\mathrm{IN}}$ both tend to one, and although the GTA method remains much more efficient than the lambda method, it too requires an increasing amount of work per time step due to the increasingly slow convergence of Eq. (4.10). Thus, various ways should be considered to accelerate this convergence using standard methods. In one-dimensional geometries, DSA [2] is a possible option which, for the grey equation, is relatively easy to implement. The boundary projecton acceleration (BPA) method [15] is another option which has the attractive feature that for linear discontinuous-type differencing schemes, $\rho_{I N} \rightarrow 0$ as $\Delta x \rightarrow \infty$. Therefore, the inner iterations will converge very rapidly for optically thick meshes, which are commonplace in thermal radiative transfer problems. Other possibilities are overrelaxation or Chebyschev acceleraton $[4,16]$, although these methods require the iteration matrix for the discretized problem to have eigenvalues that satisfy certain conditions, and it is not known even in simple geometries whether these conditions are met. These comments also apply to the conjugate gradient method [16]. Yet another possibility is the two-step acceleration method [17], which is extremely simple and conceptually applicable in any geometrical setting, although the speedups are modest (a factor of about 4.6 for the $S_{8}$ quadrature set).

A third possible way to improve the GTA method is to attempt to accelerate outer iterations using a standard acceleration method, such as overrelaxation or Chebyschev. As before, one must have some knowledge of the outer iteration eigenvalues, and to a certain extent these will be determined by any algorithm employed to accelerate the inner iterations if Eq. (4.10) is not iterated to full convergence. Another possible way to accelerate the outer iterations is to imbed extra steps in the GTA method (Eqs. (4.8)-(4.11)) which include a space-energy ADI-like treatment similar to that proposed by Axelrod, Dubois, and Rhoades [18] for the flux-limited radiation diffusion equations. In this method, which has been analyzed by Morel [19], one group-collapses the multifrequency diffusion equation into a grey diffusion equation and then alternates the solution of this space-dependent grey diffusion equation (with energy-dependence built into the residual terms) and an infinite-medium, multifrequency equation in each spatial cell (with spacedependence built into the residual terms). However, because of certain mathematical details, this idea may be better suited for diffusion than for transport problems.

In conclusion, we have presented in this aricle a new iterative GTA method for radiative transfer problems which is robust, very simple to implement, and applicable in arbitrary geometrical settings. Improvements in this method using standard or possibly novel acceleration techniques are almost surely possible and will be the subject of future research.

\section{ACKNOWLEDGMENTS}

Most of this work was performed at the Los Alamos National Laboratory, under the auspices of the U.S. Department of Energy. I would like to thank J. E. Morel for many helpful discussions, and M. L. Adams for reading the manuscript and making some useful suggestions. 


\section{REFERENCES}

1. J. A. Fleck, Jr. and J. D. Cummings, J. Comput. Phys. 8, 313 (1971).

2. R. E. Alcouffe, B. A. Clark, And E. W. LarSen, in Multiple Time Scales, edited by J. U. Brackbill and B. Cohen (Academic Press, Orlando, FL, 1985), p. 73.

3. L. H. Auve, Radiation transfer, in Rev. Comput. Phys., in press.

4. R. E. AlCOUFFe, Nucl. Sci. Eng. 64, 344 (1977).

5. E. W. LARSEN, in Proceedings, American Nuclear Saciety Topical Meeting, Advances in Reactor Computations, March 28-30, 1983, Salt Lake City 2, p. 705; Transp. Theory Stat. Phys. 13, 107 (1984).

6. G. I. Bell and S. Glasstone, Nuclear Reactor Theory (Van Nostrand-Reinhold, New York, 1970).

7. E. E. Lewis AND W. F. MilleR, JR., Computational Methods of Neutron Transport (WileyInterscience, New York, 1984).

8. C. M. LUND AND J. R. WiLson, Lawrence Livermore National Laboratory Report UCRL-84678, July 1980 (unpublished).

9. J. E. Morel, E. W. Larsen, and M. K. Matzen, J. Quant. Spectrosc. Radiat. Transfer 34, 243 (1985).

10. G. C. Pomraning, The Equations of Radiation Hydrodynamics (Pergamon, Oxford, 1973).

11. D. Mihalas and B. W. Mihalas, Foundations of Radiation Hydrodynamics (Oxford Univ. Press, New York, 1984).

12. T. R. HiLL, Los Alamos Scientific Laboratory Report LA-5990-MS, June 1975 (unpublished).

13. E. W. LARSEN, in Radiation Transport; April 1, 1986-June 30, 1986, edited by R. D. O'Dell and A. Nagy, Los Alamos National Laboratory Report LA-10844-PR, January 1987 (unpublished).

14. E. W. LARSEN, in Radiation Transport; July 1, 1986-Sept. 30, 1986, edited by R. D. O'Dell and A. Nagy, Los Alamos National Laboratory Report LA-11030-PR, August 1987 (unpublished).

15. M. L. AdAms AND W. R. MARTIN, in Proceedings, American Nuclear Society/European Nuclear Society Topical Meeting, Advances in Reactor Physics, Mathematics and Computation, April 27-30, 1987, Paris 2, p. 579.

16. L. A. Hageman And D. M. Young, Applied Iterative Methods (Academic Press, New York, 1981).

17. E. W. Larsen and W. F. Miller, JR., Trans. Amer. Nucl. Soc. 52, 416 (1986).

18. T. S. Axelrod, P. F. Dubois, And C. E. Rhoades, JR., J. Comput. Phys. 54, 205 (1984).

19. J. E. MoreL, in Radiation Transport; Jan. 1, 1986-March 31, 1986, edited by R. D. O'Dell and A. Nagy, Los Alamos National Laboratory Report LA-10792-PR, September 1986 (unpublished). 